\title{
The effect of using approximate gametic variance covariance matrices on marker assisted selection by BLUP
}

\author{
Liviu R. TotiR ${ }^{a *}$, Rohan L. Fernando ${ }^{\text {a, b }}$, Jack C.M. DekKers ${ }^{\mathrm{a}, \mathrm{b}}$, \\ Soledad A. Fernández ${ }^{\mathrm{c}}$, Bernt GuldBRANDTSEN ${ }^{\mathrm{d}}$ \\ ${ }^{\text {a }}$ Department of Animal Science, Iowa State University, Ames, IA 50011, USA \\ ${ }^{\mathrm{b}}$ Lawrence H. Baker Center for Bioinformatics and Biological Statistics, Iowa State \\ University, Ames, IA 50011, USA \\ ${ }^{c}$ Department of Statistics, The Ohio State University, Columbus, OH 43210, USA \\ ${ }^{\mathrm{d}}$ Danish Institute of Animal Science, Foulum, Denmark
}

(Received 19 September 2002; accepted 13 May 2003)

\begin{abstract}
Under additive inheritance, the Henderson mixed model equations (HMME) provide an efficient approach to obtaining genetic evaluations by marker assisted best linear unbiased prediction (MABLUP) given pedigree relationships, trait and marker data. For large pedigrees with many missing markers, however, it is not feasible to calculate the exact gametic variance covariance matrix required to construct HMME. The objective of this study was to investigate the consequences of using approximate gametic variance covariance matrices on response to selection by MABLUP. Two methods were used to generate approximate variance covariance matrices. The first method (Method A) completely discards the marker information for individuals with an unknown linkage phase between two flanking markers. The second method (Method B) makes use of the marker information at only the most polymorphic marker locus for individuals with an unknown linkage phase. Data sets were simulated with and without missing marker data for flanking markers with 2, 4, 6, 8 or 12 alleles. Several missing marker data patterns were considered. The genetic variability explained by marked quantitative trait loci (MQTL) was modeled with one or two MQTL of equal effect. Response to selection by MABLUP using Method A or Method B were compared with that obtained by MABLUP using the exact genetic variance covariance matrix, which was estimated using 15000 samples from the conditional distribution of genotypic values given the observed marker data. For the simulated conditions, the superiority of MABLUP over BLUP based only on pedigree relationships and trait data varied between $0.1 \%$ and $13.5 \%$ for Method A, between $1.7 \%$ and $23.8 \%$ for Method B, and between $7.6 \%$ and $28.9 \%$ for the exact method. The relative performance of the methods under investigation was not affected by the number of MQTL in the model.
\end{abstract}

marker assisted selection / BLUP / gametic variance covariance matrix

\footnotetext{
* Corresponding author: 1totir@iastate.edu
} 


\section{INTRODUCTION}

As a result of extensive efforts to map quantitative trait loci (QTL), a large number of markers linked to QTL have become available for genetic evaluation. A QTL with a linked marker is referred to as a marked QTL (MQTL). Genotypes at markers linked to an MQTL can be used to model the genotypic mean and the genetic variance covariance matrix at the MQTL $[8,29]$. Thus, the effects of the marker genotypes can be included as fixed effects and the gametic effects of the MQTL as random effects in the mixed linear models used for genetic evaluation by BLUP [29]. Marker genotypes, however, affect the genotypic mean only if the markers and the MQTL are in gametic phase (linkage) disequilibrium [29].

For large pedigrees, the Henderson mixed model equations (HMME) [13] provide an efficient way to obtain BLUP. One of the requirements to obtain BLUP from HMME is to compute the inverses of the variance covariance matrices of the random effects in the model. When only pedigree and trait information are used for genetic evaluation, the inverse of the conditional variance covariance matrix of the vector of unobservable genotypic values given pedigree relationships needs to be computed. Under additive inheritance, efficient algorithms are available to invert this conditional variance covariance matrix $[12,20,21]$.

Chevalet et al. [3] provided a general method to compute the genetic variance covariance matrix at an MQTL given the pedigree and marker phenotypes. This matrix, however, has a dense inverse and, thus, cannot be computed efficiently for large pedigrees [30]. When marker genotype information is available, the conditional variance covariance matrix of the vector of gametic effects at the MQTL given marker and pedigree information, which is referred to as the gametic variance covariance matrix at the MQTL, can be constructed using a recursive algorithm [8]. This matrix has a sparse inverse and, thus, can be computed efficiently even for large pedigrees, when the parental origin of marker alleles is either known [8] or not known [14, 27, 28,30]. However, the algorithms used to invert the gametic variance covariance matrix at the MQTL yield exact results only if the marker genotypes and the linkage phase between markers are known, i.e., when the marker information is complete $[15,30]$. In large pedigrees incomplete marker information is the rule rather than the exception. Wang et al. [30] provided a formula to compute the exact gametic variance covariance matrix for incomplete marker data. The use of this formula, however, is computationally intensive and thus, not feasible for large pedigrees. For large pedigrees, when marker information is incomplete, approximations must be used. 
The objective of this study was to examine the effect of two methods of approximating the gametic variance covariance matrix on response to selection by MABLUP.

\section{METHODS}

\subsection{Notation}

Consider an MQTL (Q) closely linked to two polymorphic flanking markers ( $\mathrm{M}$ and $\mathrm{N}$ ). $\mathrm{M}$ and $\mathrm{N}$ are assumed to be in linkage equilibrium with $\mathrm{Q}$ and with each other. The following diagram shows the chromosomal segments containing $\mathrm{Q}, \mathrm{M}$, and $\mathrm{N}$, for individual $i$ with parents $d$ and $s$, and for another individual $j$.

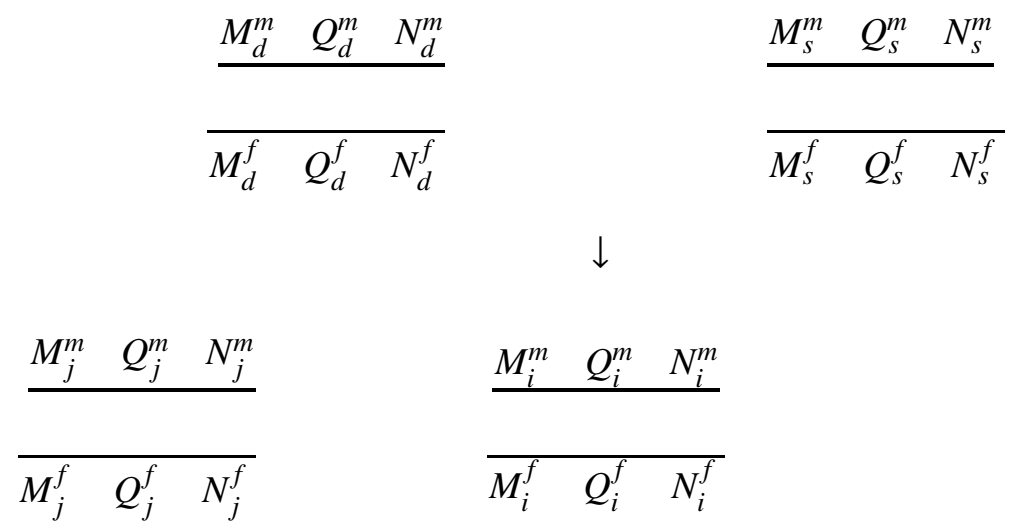

The paternal allele at a given locus is denoted by a superscript $f$, and the maternal allele by a superscript $m$. The genotypes at markers $\mathrm{M}$ and $\mathrm{N}$ may be observed, and thus, may be used for marker assisted genetic evaluation (MAGE). The genotypes at the MQTL (Q), however, cannot be observed. As discussed later, even if the marker genotypes are known, it is not always possible to infer the linkage phase between them.

The conditional covariance of the additive effects $v_{i}^{k_{i}}$ and $v_{j}^{k_{j}}$ of MQTL alleles $Q_{i}^{k_{i}}$ and $Q_{j}^{k_{j}}$ in individuals $i$ and $j$, given the observable marker information $\left(G_{o b s}\right)$, is written as

$$
\operatorname{Cov}\left(v_{i}^{k_{i}}, v_{j}^{k_{j}} \mid G_{o b s}\right)=\operatorname{Pr}\left(Q_{i}^{k_{i}} \equiv Q_{j}^{k_{j}} \mid G_{o b s}\right) \sigma_{v}^{2},
$$

where $k_{i}$ and $k_{j}$ are $m$ or $f$ if the MQTL allele origin is known [8], and 1 or 2 if the MQTL allele origin is not known [30]; $\operatorname{Pr}\left(Q_{i}^{k_{i}} \equiv Q_{j}^{k_{j}} \mid G_{\text {obs }}\right)$ is the conditional probability that $Q_{i}^{k_{i}}$ is identical by descent (IBD) to $Q_{j}^{k_{j}}$ given $G_{o b s}$; $\sigma_{v}^{2}$ is half of the variance of the additive effect of the MQTL. 


\subsection{IBD probabilities at the MQTL}

Given pedigree information, recursive formulae have been widely used to compute IBD probabilities [2,4,6,9, 18,22-25]. These formulae are based on the principle that a priori the allele transmitted from a parent to an offspring is equally likely to be the parent's maternal or paternal allele. Thus, the unconditional probability that $Q_{i}^{m}$, for example, is IBD to $Q_{j}^{k_{j}}$ can be written as

$$
\operatorname{Pr}\left(Q_{i}^{m} \equiv Q_{j}^{k_{j}}\right)=\frac{1}{2} \operatorname{Pr}\left(Q_{d}^{m} \equiv Q_{j}^{k_{j}}\right)+\frac{1}{2} \operatorname{Pr}\left(Q_{d}^{f} \equiv Q_{j}^{k_{j}}\right)
$$

When genotype information is available at a single marker, but the parental origin of the marker alleles is not known, following Wang et al. [30], the conditional probability that $Q_{i}^{k_{i}}$ is IBD to $Q_{j}^{k_{j}}$ given $G_{o b s}$ for $i \neq j$, can be written as

$$
\begin{aligned}
& \operatorname{Pr}\left(Q_{i}^{k_{i}} \equiv Q_{j}^{k_{j}} \mid G_{o b s}\right)= \\
& \operatorname{Pr}\left(Q_{i}^{k_{i}} \leftarrow Q_{d}^{1}, Q_{d}^{1} \equiv Q_{j}^{k_{j}} \mid G_{o b s}\right)+\operatorname{Pr}\left(Q_{i}^{k_{i}} \leftarrow Q_{d}^{2}, Q_{d}^{2} \equiv Q_{j}^{k_{j}} \mid G_{o b s}\right) \\
& +\operatorname{Pr}\left(Q_{i}^{k_{i}} \leftarrow Q_{s}^{1}, Q_{s}^{1} \equiv Q_{j}^{k_{j}} \mid G_{o b s}\right)+\operatorname{Pr}\left(Q_{i}^{k_{i}} \leftarrow Q_{s}^{2}, Q_{s}^{2} \equiv Q_{j}^{k_{j}} \mid G_{o b s}\right),
\end{aligned}
$$

where for example, $\operatorname{Pr}\left(Q_{i}^{k_{i}} \leftarrow Q_{d}^{1}, Q_{d}^{1} \equiv Q_{j}^{k_{j}}\right)$ denotes the probability of the event that $Q_{i}^{k_{i}}$ descended from $Q_{d}^{1}$ and $Q_{d}^{1}$ is IBD to $Q_{j}^{k_{j}}$. Note that if the parental origin of the marker allele is known, two of the four terms in equation (3) will be null. Thus, for example, for $k_{i}=m$ equation (3) becomes

$$
\begin{aligned}
& \operatorname{Pr}\left(Q_{i}^{m} \equiv Q_{j}^{k_{j}} \mid G_{o b s}\right)= \\
& \quad \operatorname{Pr}\left(Q_{i}^{m} \leftarrow Q_{d}^{m}, Q_{d}^{m} \equiv Q_{j}^{k_{j}} \mid G_{o b s}\right)+\operatorname{Pr}\left(Q_{i}^{m} \leftarrow Q_{d}^{f}, Q_{d}^{f} \equiv Q_{j}^{k_{j}} \mid G_{o b s}\right) .
\end{aligned}
$$

If the marker genotypes of $d$ and $s$ are known and $j$ is not a direct descendant of $i$, the descent of allele $Q_{i}^{k_{i}}$ from one of the alleles of $d$ or $s$, is independent of the event that alleles in $j$ are identical by descent to alleles in $d$ or $s$ [30]. 
As a result, equation (3) becomes

$$
\begin{aligned}
\operatorname{Pr}\left(Q_{i}^{k_{i}} \equiv Q_{j}^{k_{j}} \mid G_{o b s}\right)= & \operatorname{Pr}\left(Q_{i}^{k_{i}} \leftarrow Q_{d}^{1} \mid G_{o b s}\right) \operatorname{Pr}\left(Q_{d}^{1} \equiv Q_{j}^{k_{j}} \mid G_{o b s}\right) \\
& +\operatorname{Pr}\left(Q_{i}^{k_{i}} \leftarrow Q_{d}^{2} \mid G_{o b s}\right) \operatorname{Pr}\left(Q_{d}^{2} \equiv Q_{j}^{k_{j}} \mid G_{o b s}\right) \\
& +\operatorname{Pr}\left(Q_{i}^{k_{i}} \leftarrow Q_{s}^{1} \mid G_{o b s}\right) \operatorname{Pr}\left(Q_{s}^{1} \equiv Q_{j}^{k_{j}} \mid G_{o b s}\right) \\
& +\operatorname{Pr}\left(Q_{i}^{k_{i}} \leftarrow Q_{s}^{2} \mid G_{o b s}\right) \operatorname{Pr}\left(Q_{s}^{2} \equiv Q_{j}^{k_{j}} \mid G_{o b s}\right),
\end{aligned}
$$

where for example, $\operatorname{Pr}\left(Q_{i}^{k_{i}} \leftarrow Q_{d}^{1} \mid G_{o b s}\right)$ denotes the probability of descent of $Q_{i}^{k_{i}}$ from $Q_{d}^{1}$ (PDQ). Note that if the parental origin at the marker allele is known, for example $k_{i}=m$, equation (5) becomes

$$
\begin{aligned}
\operatorname{Pr}\left(Q_{i}^{m} \equiv Q_{j}^{k_{j}} \mid G_{o b s}\right)=\operatorname{Pr} & \left(Q_{i}^{m} \leftarrow Q_{d}^{m} \mid G_{o b s}\right) \operatorname{Pr}\left(Q_{d}^{m} \equiv Q_{j}^{k_{j}} \mid G_{o b s}\right) \\
& +\operatorname{Pr}\left(Q_{i}^{m} \leftarrow Q_{d}^{f} \mid G_{o b s}\right) \operatorname{Pr}\left(Q_{d}^{f} \equiv Q_{j}^{k_{j}} \mid G_{o b s}\right) .
\end{aligned}
$$

When marker information for the parents is missing, the independence required to obtain equation (5) from equation (3) may not hold true [30]. Thus, equation (5) may yield only approximate results when marker information is missing. When the parental origin at the marker genotype is not known, equation (5) cannot be used directly to compute IBD probabilities within an individual $(i=j)$ [30]. For this situation, IBD probabilities can be computed using formula (11) in Wang et al. [30].

When genotype information is available at markers flanking the MQTL, the conditional probability that $Q_{i}^{k_{i}}$ is IBD to $Q_{j}^{k_{j}}$ given $G_{o b s}$ for $i \neq j$, can be obtained from (5) but with PDQ computed conditional on the flanking marker information [10]. In this situation, even when marker genotypes are observed, if the linkage phase between the two flanking markers is not known, the independence required to obtain equation (5) from equation (3) may not hold true [15]. Thus, equation (5) may yield only approximate results when the linkage phase between flanking markers is not known.

For a single marker, Wang et al. [30] derived formulae for computing PDQ in terms of recombination rates and probabilities of descent for a marker allele (PDM), e.g. $\operatorname{Pr}\left(M_{i}^{k_{i}} \leftarrow M_{d}^{1} \mid G_{o b s}\right)$. When some marker genotypes are missing, however, computing the required PDM may be computationally intensive. For example, when marker information is missing for an individual $i$ and its 
parents $d$ and $s$, the $\operatorname{PDM} \operatorname{Pr}\left(M_{i}^{1} \leftarrow M_{d}^{1} \mid G_{o b s}\right)$ can be written as

$$
\begin{aligned}
\operatorname{Pr}\left(M_{i}^{1} \leftarrow M_{d}^{1} \mid G_{o b s}\right)= & \\
& \sum_{G_{d}} \sum_{G_{s}} \sum_{G_{i}} \operatorname{Pr}\left(M_{i}^{1} \leftarrow M_{d}^{1} \mid G_{d}, G_{s}, G_{i}\right) \operatorname{Pr}\left(G_{d}, G_{s}, G_{i} \mid G_{o b s}\right) .
\end{aligned}
$$

In equation (7), the calculation of $\operatorname{Pr}\left(G_{d}, G_{s}, G_{i} \mid G_{o b s}\right)$ can be computationally demanding for a pedigree with a large number of missing marker genotypes. Thus, to make computations feasible for large pedigrees with many missing marker genotypes, $\operatorname{Pr}\left(G_{d}, G_{s}, G_{i} \mid G_{o b s}\right)$ must also be approximated. Note that when flanking markers are used, PDM are replaced by probabilities of descent of a haplotype [11]. Again, when the linkage phase between the flanking markers is not known, these probabilities must be approximated.

If the gametic variance covariance matrix is constructed using the recursive formula (5), then its inverse can also be obtained using a simple recursive formula $[27,30]$. But, for large pedigrees with many missing markers, this requires the efficient computation of approximate PDQ. In the next section we discuss two strategies to compute approximate PDQ for large pedigrees given genotypes at two flanking markers.

\subsection{Approximate calculations of PDQ probabilities}

The genotype at a marker locus may be unobserved (missing) or observed. Based on the observable marker data for the entire pedigree, some of the unobserved marker genotypes can be inferred with certainty. In this paper, the genotype elimination algorithm by Lange and Goradia [17] was applied to the entire pedigree. This algorithm yields a list of possible genotypes for each of the unobserved genotypes. Whenever such a list contains only one possible genotype, the unobserved genotype is inferred with certainty and is treated as an observed genotype. An observed genotype is ordered if the parental origin of the alleles is known, or unordered if the parental origin is unknown.

One simple method to compute PDQ is to use marker information only when the genotypes are ordered at both flanking markers, i.e., when the linkage phase between the markers is known. In this case, PDQ can be computed as described by Goddard [10]. For example, if we assume at most a single recombination between the flanking markers, the PDQ for MQTL allele $Q_{i}^{m}$, conditional on the maternal marker haplotype inherited by $i$, can be calculated as shown in Table I. The PDQ for MQTL allele $Q_{i}^{f}$, conditional on the paternal marker haplotype inherited by $i$, can be calculated in a similar manner.

When the phase is not known, marker information is completely ignored, and thus, the PDQ for each of the parental alleles is equal to 0.5. This method will be referred to as Method A. 
Table I. Given the maternal marker haplotype inherited by $i$, the probability that the MQTL allele $Q_{i}^{m}$ descends from the parental allele $Q_{p}^{k}$ (PDQ), where $p$ is $d$ or $s$ and $k$ is $m$ or $f . M_{d}^{?} \quad N_{d}^{?}$ denotes an unknown haplotype. Here $r_{1}$ is the recombination rate between marker locus $M$ and MQTL $Q ; r_{2}$ is the recombination rate between marker locus $N$ and MQTL $Q$.

\begin{tabular}{llllll}
\hline Haplotype & \multicolumn{5}{c}{$Q_{p}^{k}$} \\
\cline { 2 - 6 } inherited & $Q_{d}^{m}$ & $Q_{d}^{f}$ & $Q_{s}^{m}$ & $Q_{s}^{f}$ \\
\hline$M_{d}^{m}$ & $N_{d}^{m}$ & 1.0 & 0.0 & 0.0 & 0.0 \\
$M_{d}^{m}$ & $N_{d}^{f}$ & $\frac{r_{2}}{r_{1}+r_{2}}$ & $\frac{r_{1}}{r_{1}+r_{2}}$ & 0.0 & 0.0 \\
$M_{d}^{f}$ & $N_{d}^{m}$ & $\frac{r_{1}}{r_{1}+r_{2}}$ & $\frac{r_{2}}{r_{1}+r_{2}}$ & 0.0 & 0.0 \\
$M_{d}^{f}$ & $N_{d}^{f}$ & 0.0 & 1.0 & 0.0 & 0.0 \\
$M_{d}^{?}$ & $N_{d}^{?}$ & 0.5 & 0.5 & 0.0 & 0.0 \\
\hline
\end{tabular}

An alternative method that makes better use of the marker information is described below. This alternative method will be referred to as Method B. As in Method A, when the linkage phase between the markers is known, PDQ can be computed conditional on marker haplotypes [10]. When the linkage phase between the markers is not known, genotype information at one of the two flanking markers can be used to compute PDQ $[19,26]$. The genotype at the marker locus may be ordered or unordered, and these two cases are considered separately. When the marker genotype is ordered, PDQ can be computed as described by Fernando and Grossman [8]. For example, the PDQ for the MQTL allele $Q_{i}^{m}$, conditional on the maternal marker allele inherited by $i$, can be calculated as shown in Table II. The PDQ for MQTL allele $Q_{i}^{f}$, conditional on the paternal marker allele inherited by $i$, can be calculated in a similar manner.

When marker genotypes of an offspring are unordered, marker information can be ignored $[8,19]$. However, as discussed later, this results in a loss of information. The genotype of an offspring at a marker locus may be unordered only if it is heterozygous at that locus. Given that the genotype of an individual is heterozygous, it will be unordered if both its parents are heterozygous for the same alleles, or one of the parents is heterozygous for the same alleles while the marker information at the other parent is missing, or if the marker information is missing in both parents. When the marker genotype is unordered, PDQ can be calculated as described by Wang et al. [30] by multiplying a $2 \times 4$ matrix of PDM by a $4 \times 4$ matrix involving recombination rates. If the marker genotypes are observed for both parents, the PDM are easily obtained from formula (A1) in Wang et al. [30]. For example, when both parents and the offspring have marker genotype $A_{1} A_{2}$, the PDM for marker allele $M_{i}^{1}$ are given in row one of Table III. 
Table II. Given the maternal marker allele inherited by $i$, the probability that MQTL allele $Q_{i}^{m}$ descends from the parental allele $Q_{p}^{k}$ (PDQ), where $p$ is $d$ or $s$ and $k$ is $m$ or $f . M_{d}^{\text {? }}$ denotes unknown descent. Here $r_{1}$ is the recombination rate between marker locus $M$ and MQTL $Q$.

\begin{tabular}{ccccc}
\hline Allele & \multicolumn{4}{c}{$Q_{p}^{k}$} \\
\cline { 2 - 5 } inherited & $Q_{d}^{m}$ & $Q_{d}^{f}$ & $Q_{s}^{m}$ & $Q_{s}^{f}$ \\
\hline$M_{d}^{m}$ & $1-r_{1}$ & $r_{1}$ & 0.0 & 0.0 \\
$M_{d}^{f}$ & $r_{1}$ & $1-r_{1}$ & 0.0 & 0.0 \\
$M_{d}^{?}$ & 0.5 & 0.5 & 0.0 & 0.0 \\
\hline
\end{tabular}

Table III. Given the parental marker information, the probability that marker allele $M_{i}^{1}$ descends from the parental allele $M_{p}^{k}(\mathrm{PDM})$, where $p$ is $d$ or $s$ and $k$ is 1 or 2. - denotes missing marker information.

\begin{tabular}{ccccccc}
\hline \multicolumn{3}{c}{ Genotype of } & \multicolumn{5}{c}{$M_{p}^{k}$} \\
\cline { 4 - 7 } $\mathrm{d}$ & $\mathrm{s}$ & $\mathrm{i}$ & $M_{d}^{1}$ & $M_{d}^{2}$ & $M_{s}^{1}$ & $M_{s}^{2}$ \\
\hline$A_{1} A_{2}$ & $A_{1} A_{2}$ & $A_{1} A_{2}$ & 0.5 & 0.0 & 0.5 & 0.0 \\
$A_{1} A_{2}$ & - & $A_{1} A_{2}$ & 0.5 & 0.0 & 0.25 & 0.25 \\
- & - & $A_{1} A_{2}$ & 0.25 & 0.25 & 0.25 & 0.25 \\
\hline
\end{tabular}

When marker genotypes are missing in the parents, Wang et al. [30] used equation (7) to compute the PDM. But, this can be computationally demanding in large pedigrees with many missing genotypes. Thus, we compute the PDM using only the marker genotypes that are observed in the parents. For example, if the marker genotype is missing in parent $s$, and is $A_{1} A_{2}$ for $d$ and $i$, the PDM for marker allele $M_{i}^{1}$, ignoring all the other marker information in the pedigree, are given in row two of Table III. Row three of Table III gives the PDM for marker allele $M_{i}^{1}$, ignoring all the other marker information in the pedigree, for the case when the marker genotype is missing for $d$ and $s$, and is $A_{1} A_{2}$ in $i$. Thus, when marker genotypes of an offspring are unordered, PDM of the type described above can be computed easily. As mentioned earlier for Method A, when the genotypes at both markers are unobserved, the PDQ for each of the parental alleles is equal to 0.5 .

It is important to note that, under the assumption of at most a single recombination between flanking markers, some PDQ are equal to one (Tab. I). When this occurs, the MQTL allele $Q_{i}^{m}$, for example, is traced with certainty to MQTL allele $Q_{d}^{m}$, and thus, $\operatorname{Pr}\left(Q_{i}^{m} \equiv Q_{d}^{m} \mid G_{o b s}\right)=1$. A similar situation will occur when, for example, $\operatorname{Pr}\left(Q_{d}^{m} \equiv Q_{d}^{f} \mid G_{o b s}\right)=1$. Recall that $Q_{i}^{m}$ is either $Q_{d}^{m}$ or $Q_{d}^{f}$. Thus, regardless of the value of the $\operatorname{PDQ}, \operatorname{Pr}\left(Q_{i}^{m} \equiv Q_{d}^{m}\right.$ । $\left.G_{o b s}\right)=\operatorname{Pr}\left(Q_{i}^{m} \equiv Q_{d}^{f} \mid G_{o b s}\right)=1$. When the IBD probability between any pair 
of MQTL alleles is one, the gametic variance covariance matrix will not be positive definite. To avoid this problem, if two alleles are IBD with a probability of one, only the effect of one of these two alleles is included in the mixed linear model. A side effect of this approach is the reduction in the number of equations in HMME and thus, an increase in the computational efficiency [10].

\subsection{Calculation of the inverse of the gametic variance covariance matrix}

The PDQ computed as described above can be used in formulae (18), (19), and (21) of Wang et al. [30] to efficiently obtain the inverse of the gametic variance covariance matrix. Formula (19) of Wang et al. [30] requires computing the IBD probabilities between the MQTL alleles of the parents. These were computed using the recursive formula (5), except for alleles within an individual with unordered markers. For individuals with unordered markers, IBD probabilities between their maternal and paternal alleles were computed using formula (11) in [30].

Recursive computation of the IBD probability between any pair of alleles may require IBD probabilities previously used in computing the IBD probability between other pairs of alleles. Thus, as in Abdel-Azim and Freeman [1], in order to avoid computing the same IBD probability repeatedly, upon the computation of an IBD probability it was stored for possible future use. While Abdel-Azim and Freeman [1] used linked lists to store the probabilities, we used a map container class of the $\mathrm{C}++$ Standard Template Library. Each data item (an IBD probability in this case) stored in a map container class is indexed by a key. For elements $i$ and $j$ of the IBD matrix, $i$ and $j$ were used as the key to store and retrieve this element.

\subsection{Estimation of the exact genetic variance covariance matrix by MCMC}

ESIP, an MCMC sampler that combines the Elston-Stewart algorithm with iterative peeling [7], was used to sample the genotypes for unobserved markers and all the MQTL genotypes jointly from the entire pedigree. Given the genotypic effects and the sampled MQTL genotypes, a vector of genotypic values was obtained for the pedigree. The genetic variance covariance matrix was estimated from 15000 independently distributed vectors of genotypic values. A scenario with 50000 vectors of genotypic values was also considered (Sect. 3.1). To validate this approach, the genetic variance covariance matrix estimated by ESIP was compared with the exact genetic variance covariance matrix calculated by using formula (27) of Wang et al. [30] for the case of a single marker linked to the MQTL. 


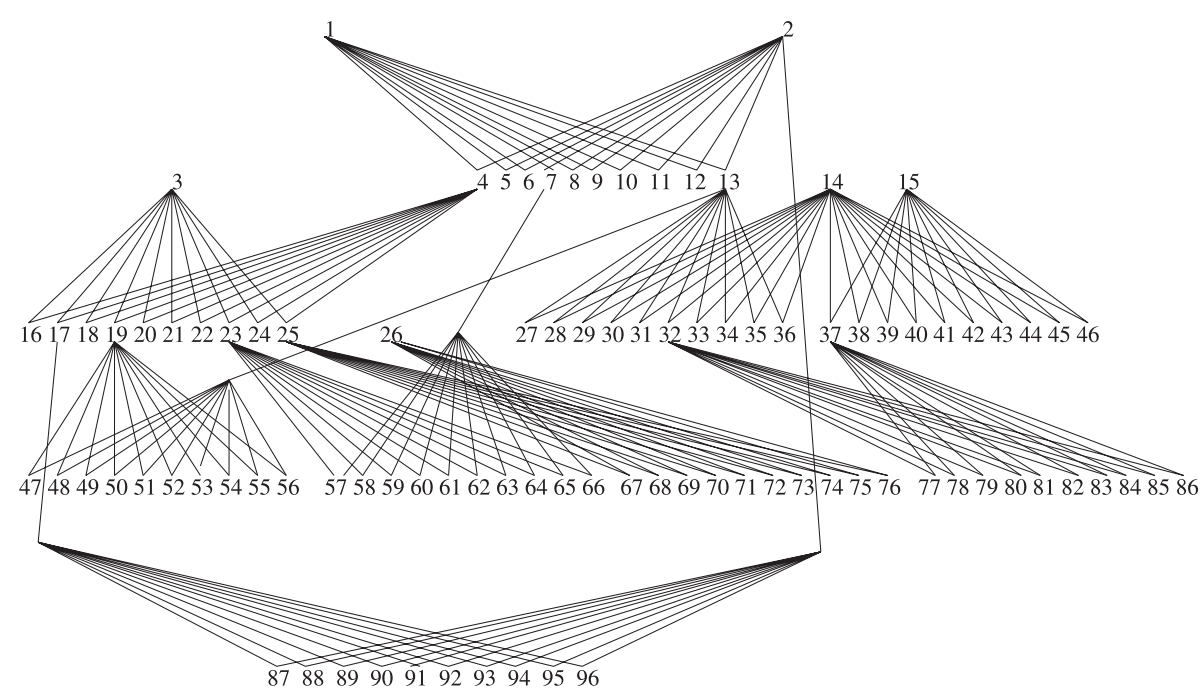

Figure 1. Pedigree used.

\subsection{Simulation study}

Simulated data were used to examine the consequences of using approximate gametic covariance matrices on response to selection by MABLUP. Trait phenotypes and genotypes at two markers flanking the MQTL were simulated for the hypothetical pedigree shown in Figure 1. This pedigree spans four generations, has 96 individuals, several loops, and each of its nuclear families has 10 offspring. In all simulations, the recombination rate between each of the flanking markers and the MQTL was 0.05 .

To identify the differences between the two approximations considered, we simulated experimental situations for which the use of marker information is expected to have a large effect on response to selection. Thus, a trait with a heritability of 0.1 that was not measured on the candidates for selection (individuals 47 to 96) was simulated. To make the simulation computationally manageable, only one MQTL was simulated to account for $28.5 \%$ of the total genetic variance (2.85\% of the phenotypic variance) for all but one of the experimental situations considered. In addition to the MQTL, the trait was determined by 40 identical, unlinked, biallelic QTL with an allele frequency of 0.5 .

To examine the effect of the number of marker alleles $\left(N_{a}\right)$ on the approximations, simulation results were obtained for the models without missing marker genotypes, one MQTL, and with $N_{a}=2,4,6,8$, or 12 at each of the flanking markers. A frequency of $\frac{1}{N_{a}}$ was used for each allele. 
To examine if the number of MQTL included in the model has an effect on the approximations, simulation results were obtained for models without missing marker genotypes, $N_{a}=2$ at each of the flanking markers, and with either one MQTL or two MQTL, with each of the two MQTL accounting for $14.25 \%$ of the total genetic variance.

To examine the effect of missing marker data on the approximations, four missing marker data patterns were considered. In the first missing marker data pattern (P1), founders 1, 2, 3, 14, and 15 had missing marker genotypes on both flanking markers. In the second pattern (P2), founders $1,2,3,14,15$, and half of the terminal offspring that are not candidates for selection had missing marker genotypes on both flanking markers. In the third pattern (P3), founders $1,2,3,14,15$, and all terminal offspring that are not candidates for selection had missing marker genotypes on both flanking markers. Finally, in the fourth pattern considered (P4), all parents were genotyped but all terminal offspring that are not candidates for selection had missing marker genotypes on both flanking markers. Simulation results were obtained for the models with one MQTL, $N_{a}=2$ for missing patterns P1, P2, P3, and P4.

For each experimental situation considered, the 5 highest ranking individuals were selected out of the 50 candidates for selection based on genetic evaluations obtained by: BLUP using only phenotypic data, MABLUP using the gametic variance covariance matrix calculated by Method A, MABLUP using the gametic variance covariance matrix calculated by Method B, and MABLUP using the exact genetic variance covariance matrix estimated by ESIP (Method E). Response to selection obtained by BLUP using only phenotypic data was used as the reference value to compare methods A, B and E. For each of the three methods under investigation, the percent superiority in response by MABLUP over response by BLUP was calculated as

$$
\frac{R_{M A B L U P}-R_{B L U P}}{R_{B L U P}} \times 100,
$$

where, for example, $R_{M A B L U P}$ is the difference between the mean of the genotypic values of the candidates selected using MABLUP and the mean of the genotypic values of the candidates for selection.

\section{RESULTS}

\subsection{Validation of the use of ESIP to estimate the exact genetic variance covariance matrix}

To validate ESIP as an accurate method to estimate the exact genetic variance covariance matrix, we used two simulated data sets with $N_{a}=4$ at each of the flanking markers, one with no missing marker data and one with a missing 
Table IV. Maximum, mean and standard deviation of the elements of the matrices of absolute differences between the exact and the estimated genetic variance covariance matrices, for the situations with no missing marker data and with missing pattern P1 for $N_{a}=4$. The estimated matrices were calculated using 15000 or 50000 samples generated by ESIP.

\begin{tabular}{ccccc}
\hline Marker data & No. of samples & Maximum & Mean & S.D. \\
\hline no missing & 15000 & $7.0 \times 10^{-2}$ & $1.3 \times 10^{-2}$ & $1.0 \times 10^{-2}$ \\
& 50000 & $3.0 \times 10^{-2}$ & $6.7 \times 10^{-3}$ & $5.0 \times 10^{-3}$ \\
\hline pattern P1 & 15000 & $6.4 \times 10^{-2}$ & $1.3 \times 10^{-2}$ & $1.0 \times 10^{-2}$ \\
& 50000 & $3.2 \times 10^{-2}$ & $6.7 \times 10^{-3}$ & $5.0 \times 10^{-3}$ \\
\hline
\end{tabular}

marker data pattern P1. However, because it is not computationally feasible to use formula (27) from Wang et al. [30] to calculate the exact genetic variance covariance matrix given the information at flanking markers with missing genotypes, the marker data at one of the two marker loci was discarded. For each of the two situations considered, the exact genetic variance covariance matrix was calculated using formula (27) of Wang et al. [30], and it was estimated using 15000 or 50000 independently distributed vectors of genotypic values sampled by ESIP. The absolute difference between the exact genetic variance covariance matrix and the estimated variance covariance matrix was calculated for each of the two situations considered. For each matrix of absolute differences, the maximum, mean, and standard deviation of its elements were computed, and are reported in Table IV.

These statistics were used to assess the accuracy of the variance covariance matrix estimated by ESIP. For both situations, the accuracy of the genetic variance covariance matrix estimated using 15000 samples was considered sufficient.

\subsection{Comparison of response to selection obtained with different MABLUP methods}

The running mean of percent superiority of MABLUP by methods A, B, and $\mathrm{E}$ was calculated based on 5000 replicates for each experimental situation considered. After about 3000 replicates each running mean was virtually constant.

\subsubsection{Response to selection for situations with no missing marker information}

Figure 2 summarizes the percent superiority of MABLUP estimated from 5000 replicates of the simulation, by methods $\mathrm{A}, \mathrm{B}$, and $\mathrm{E}$, and by the number of alleles at a marker when marker data were available for all individuals. 


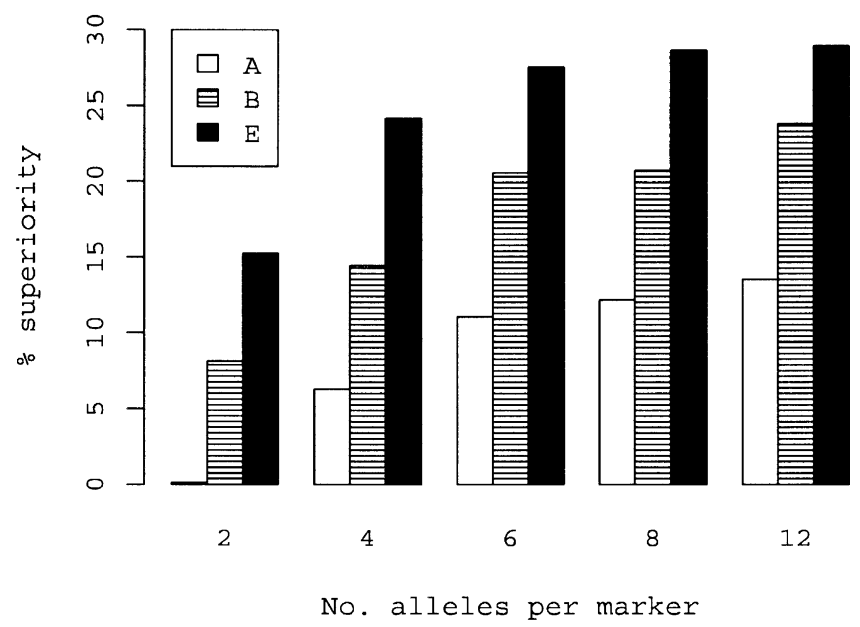

Figure 2. The summary of percent superiority of MABLUP estimated from 5000 replicates of the simulation, by methods A, B, and E, and by the number of alleles at each of the flanking markers when all individuals have marker data.

Method A performed worst in all situations. Compared with Method A, Method B resulted in an increased response to selection for all situations considered. The difference in superiority obtained with Method B versus the superiority obtained with Method A was approximately 8 to $10.3 \%$.

It can be seen from Figure 2 that as $N_{a}$ increases, the percent superiority of MABLUP by both methods A and B increases. However, the increase for method A is more rapid, especially from $N_{a}=2$ to $N_{a}=4$. Recall that in Method A marker information in a parent is used only if the markers are doubly heterozygous and their linkage phase is known. In contrast for Method B, even when only one of the two markers is heterozygous and even if the linkage phase is not known marker information in a parent may be used. In both methods the increase in percent superiority is due to the increase in the number of heterozygous genotypes at the two marker loci. A possible explanation for the more rapid change for Method A is that for the situation with $N_{a}=2$ most individuals were evaluated using only pedigree and trait information. When the number of alleles increased to 4 , markers became informative for many of these individuals. In contrast for Method B, even with $N_{a}=2$ many individuals were evaluated using pedigree, trait and marker information at one locus. When the number of alleles increases to 4 , many individuals that were originally evaluated using information at one marker are evaluated using information at both markers. The change in percent superiority of MABLUP 


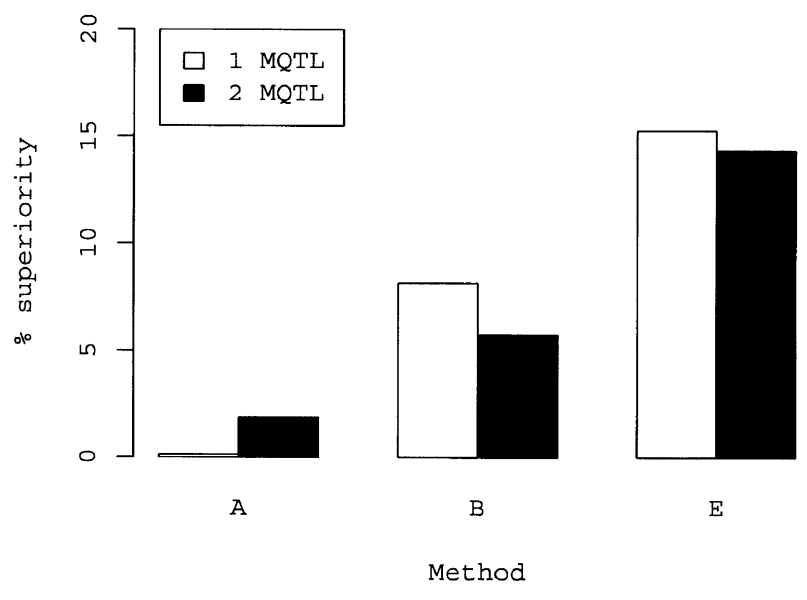

Figure 3. The summary of percent superiority of MABLUP estimated from 5000 replicates of the simulation, by methods $\mathrm{A}, \mathrm{B}$, and $\mathrm{E}$, and by the number of MQTL in the model ( 1 or 2 ) when all individuals have marker data and each of the flanking markers has two alleles.

is higher when going from using no marker information to using two markers, which is what happens with Method A, than when going from using one marker to using two markers, which is what happens with Method B.

Figure 2 also shows the effect of the increase in the number of alleles at the two marker loci on percent superiority of MABLUP by Method E. Again, the increase in the number of heterozygous genotypes at the marker loci resulted in an increase in superiority, for example, by $\sim 8.9 \%$ when moving from $N_{a}=2$ to $N_{a}=4$. However, the rate of increase is higher for Method B than for Method E. A possible explanation for this result is that Method E always uses information at both marker loci, where phase information comes from the entire pedigree. Thus, even with $N_{a}=2$ all informative markers are used. For Method E, the increase in percent response is due only to the increase in the number of informative markers. Figure 2 also shows that, while for methods A and B the percent superiority increased continuously as $N_{a}$ increased from two to 12 , for method $\mathrm{E}$ the percent superiority stayed approximately constant as $N_{a}$ increased from six to 12 .

Figure 3 shows the superiority of MABLUP for models with one MQTL or two MQTL of equal magnitude. These results indicate that the number of MQTL included in the model has little or no effect on the relative performance of the methods under investigation. 

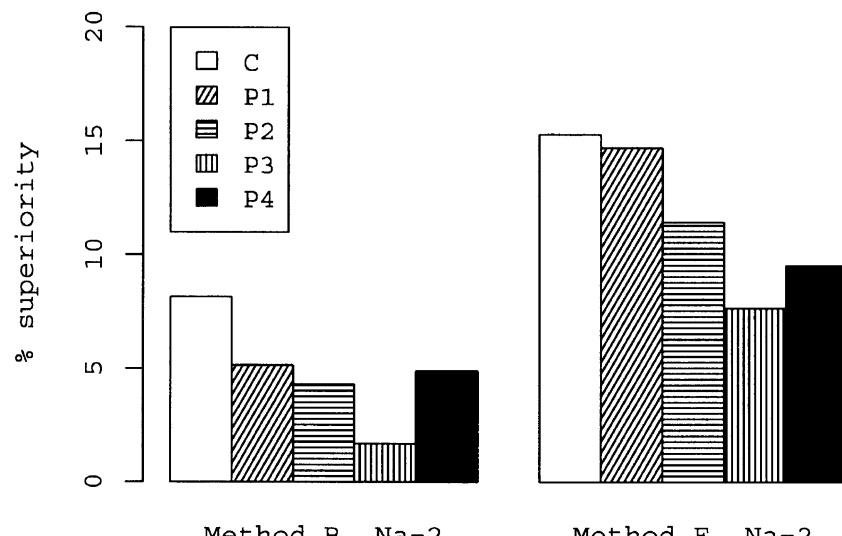

Method $\mathrm{E}, \mathrm{Na}=2$

Figure 4. The summary of percent superiority of MABLUP estimated from 5000 replicates of the simulation, by methods B and E with $N_{a}=2$, for the situation with no missing marker data (C), and for missing marker data patterns P1, P2, P3, and P4 (Sect. 2.6).

\subsubsection{Response to selection for situations with missing marker information}

Figure 4 summarizes the percent superiority of MABLUP estimated from 5000 replicates of the simulation, by methods B and E with $N_{a}=2$ for missing marker data patterns P1, P2, P3, and P4 as well as for the situation with no missing marker data (pattern $\mathrm{C}$ ).

Marker information from offspring contributes to MABLUP in two ways. First, when both parents and offspring have marker information, it may be possible to trace the inheritance of MQTL alleles from parents to some of the offspring. These offspring are said to be informative. Then, the phenotypic information from each of these informative offspring contributes to the evaluation of the MQTL effects in the parents. Second, even if the genotypes of the parents are missing, when genotype information is available on many offspring, it may be possible to infer the genotypes and phase at the flanking markers of the parents. The inference of the linkage phase will be more certain for parents that have more offspring with marker data. When the linkage phase in the parents is inferred with greater certainty, the inheritance of MQTL alleles is traced more effectively. Thus, for parents with many genotyped offspring, the contribution of the phenotypic information from each of the informative offspring becomes more effective for the evaluation of MQTL effects in the parents.

Recall that Method E is an MCMC method that makes optimal use of all the marker information from the entire pedigree. Although the marker genotypes 
for the founders were missing in P1, all their offspring were genotyped. Thus, when Method E was used, the missing marker genotypes of the founders as well as the linkage phase between the flanking markers were inferred with a high level of certainty. This explains the negligible difference in superiority between pattern $\mathrm{C}$ and pattern P1 in Figure 4. When method B was used, the superiority with pattern P1 was considerably lower than with pattern $\mathrm{C}$ (Fig. 4). This was because Method B does not make optimal use of the marker information from the offspring to infer the genotypes and linkage phase in the founders.

Recall that in addition to the founders having missing genotypes, the percentage of missing genotypes among the terminal offspring that are not candidates for selection, is $0 \%$ for $\mathrm{P} 1,50 \%$ for $\mathrm{P} 2$, and $100 \%$ for $\mathrm{P} 3$. With both Methods B and E, the loss in superiority seems to be proportional to the percentage of offspring with missing genotypes (Fig. 4).

In pattern $\mathrm{P} 4$, all parents are genotyped but all terminal offspring that are not candidates for selection are not genotyped. Thus, in both Methods B and E, only the phenotypic information of the non-terminal offspring contributes to the evaluation of the MQTL effects in the founders. Furthermore, in Method E, the linkage phase between the flanking markers is inferred only based on the marker information of the non-terminal offspring. Thus, for both Methods B and $\mathrm{E}$, the superiority with pattern $\mathrm{P} 4$ was considerably lower than that with pattern $\mathrm{C}$ (Fig. 4) where all offspring contribute with marker and phenotype information. Note that the difference in superiority between P3 and P4 is larger for Method B than for Method E. The same argument used to explain the difference in superiority between $\mathrm{C}$ and $\mathrm{P} 1$ is valid for the difference between P3 and P4.

\section{DISCUSSION}

Pong-Wong et al. [19] used a method that is similar to our Method B to compute the approximate gametic variance covariance matrix, yet with some important differences. First, when marker genotypes are missing, Pong-Wong et al. [19] used only the marker genotypes of parents and offspring to determine the missing marker genotypes. In contrast, in Method B genotype elimination [17] was used to determine the missing marker genotypes given the observable marker data for the entire pedigree. Second, Pong-Wong et al. [19] used a deterministic approach developed by Knott and Haley [16] to calculate IBD probabilities between the offspring of founders conditional on marker information of both flanking loci. When the approach proposed by Pong-Wong et al. is used, however, the inverse of the gametic variance covariance matrix cannot be obtained efficiently and thus, their approach is not useful for 
MABLUP. In Method B, to compute the PDQ for offspring from founders, we used only information from one marker locus, and all IBD probabilities were computed using the recursive formula (5). Thus, the gametic variance covariance obtained using Method B could be inverted efficiently. Finally, when the linkage phase was not known in non-founders both the method used by PongWong et al. [19] and Method B used only one of the two flanking markers to obtain IBD probabilities. However, if the genotype at the marker locus used is unordered, Pong-Wong et al. [19] ignored the marker information at this locus. In Method B, however, following Wang et al. [30], marker information was used to calculate the IBD probabilities. The benefit of using marker information in this situation is described below. Consider the covariance between the MQTL effects of half-sibs that receive different marker alleles from their sire. If the marker genotype in the sire is unordered, Pong-Wong et al. [19] used only pedigree information to compute this covariance between these half-sibs, which is equal to $\frac{1}{2} \sigma_{v}^{2}$. However, if the marker information is used as described by Wang et al. [30] the covariance between half-sibs will be $2(1-r) r \sigma_{v}^{2}$.

Methods A and B yield approximate gametic variance covariance matrices due to the following reasons. The gametic variance covariance matrix is constructed in both methods using equation (5). However, when the marker genotypes for parents are missing, or even when there is no missing marker data but the linkage phase between flanking markers is unknown, this recursive equation yields approximate IBD probabilities. Furthermore, the PDQ required to calculate IBD probabilities using equation (5) are approximated to be able to accommodate large pedigrees with many missing markers. For all situations considered, Method B yielded a higher response to selection and thus indicates a better use of the available marker information. However, the results obtained with Method E indicate that even Method B does not adequately utilize the observable marker information. For example, with missing data pattern P1 (Fig. 4) where the marker genotypes of the founders are missing, the loss in response to selection by MABLUP was negligible when Method E was used. In contrast, when Method B was used the loss in response to selection by MABLUP was considerable. This shows that when a method that makes optimal use of marker information (such as Method E) is employed, marker information from many offspring can make up for missing genotypes in the parents. However, even when marker information is available on all the parents, genotyping terminal offspring results in greater response to selection by MABLUP.

At present, in Method B PDQ are computed based only on the marker information of the individual and its parents. Method B could be improved by computing the PDQ conditional on observable marker data from all "closely" related individuals [30]. This can be done by deterministic methods such as the 
Elston-Stewart algorithm [5], or by MCMC. With MCMC methods it may be possible to estimate PDQ using all the marker information from large and complex pedigrees. This, however, would not be possible with the Elston-Stewart algorithm except for simple pedigrees.

From the comparisons in this paper it is not possible to determine how much of the loss in response is due to the violation of the independence condition that is required to obtain equation (5) as opposed to the use of approximate PDQ. The use of exact PDQ in Method B in comparison to Method E would allow us to determine the loss in response caused by the use of equation (5).

The main conclusion of this paper is that the choice of the PDQ approximations used to construct the gametic variance covariance matrix has a significant impact on response to selection by MABLUP. Furthermore we demonstrated the potential advantage of improving the current approximations of the gametic variance covariance matrix.

\section{ACKNOWLEDGEMENTS}

This journal paper of the Iowa Agriculture and Home Economics Experiment Station, Ames, Iowa, Project No. 6587, was supported by Hatch Act and State of Iowa funds. This work was partially funded by the Monsanto Company and by award No. 2002-35205-1156 of the National Research Initiative Competitive Grants Program of the USDA. Helpful comments and suggestions from the editor and two referees are gratefully acknowledged.

\section{REFERENCES}

[1] Abdel-Azim G., Freeman A.E., A rapid method for computing the inverse of the gametic covariance matrix between relatives for a marked quantitative trait locus, Genet. Sel. Evol. 33 (2001) 153-173.

[2] Chang H.L., Studies on estimation of genetic variances under non-additive gene action, Ph.D. Thesis, University of Illinois at Urbana-Champaign, 1988.

[3] Chevalet C., Gillois M., Khang J.V.T., Conditional probabilities of identity of genes at a locus linked to a marker, Génét. Sél. Évol. 16 (1984) 431-444.

[4] Cockerham C.C., Effects of linkage on the covariance between relatives, Genetics 41 (1956) 138-141.

[5] Elston R.C., Stewart J., A general model for the genetic analysis of pedigree data, Hum. Hered. 21 (1971) 523-542.

[6] Emik L.O., Terrill C.E., Systematic procedures for calculating inbreeding coefficients, J. Hered. 40 (1949) 51-55.

[7] Fernandez S.A., An algorithm to sample genotypes in complex pedigrees, $\mathrm{Ph} . \mathrm{D}$. Thesis, Iowa State University, 2001.

[8] Fernando R.L., Grossman M., Marker assisted selection using best linear unbiased prediction, Genet. Sel. Evol. 21 (1989) 467-477. 
[9] Gillois M., La relation d'identité en génétique, [genetic identity relationship], Ph.D. Thesis, Fac. Sci. Paris. (1964) in: Jacquard A. (Ed.), The Genetic Structure of Populations, Springer-Verlag, Germany, 1974.

[10] Goddard M.E., A mixed model for analysis of data on multiple genetic markers, Theor. Appl. Genet. 83 (1992) 878-886.

[11] Grignola F.E., Hoeschele I., Tier B., Mapping quantitative trait loci in outcross populations via residual maximum likelihood. I. Methodology, Genet. Sel. Evol. 28 (1996) 479-490.

[12] Henderson C.R., A simple method for computing the inverse of a numerator relationship matrix used in prediction of breeding values, Biometrics 32 (1976) 69-83.

[13] Henderson C.R., Applications of linear models in animal breeding, University of Guelph, Guelph, Ontario, Canada, 1984.

[14] Hoeschele I., Elimination of quantitative trait loci equations in an animal model incorporating genetic marker data, J. Dairy Sci. 76 (1993) 1693-1713.

[15] Hoeschele I., Mapping of quantitative trait loci in complex pedigrees, in: Balding D., Bishop M., Cannings C. (Eds.), Handbook of Statistical Genetics, Wiley, 2001, pp. 599-644.

[16] Knott S., Haley C.S., Simple multiple-marker sib-pair analysis for mapping quantitative trait loci, Heredity 81 (1998) 48-54.

[17] Lange K., Goradia T.M., An algorithm for automatic genotype elimination, Am. J. Hum. Genet. 40 (1987) 250-256.

[18] Lo L.L., Fernando R.L., Cantet R.J.C., Grossman M., Theory of modelling means and covariances in a two-breed population with dominance, Theor. Appl. Genet. 90 (1995) 49-62.

[19] Pong-Wong R., George A.W., Wooliams J.A., Haley C.S., A simple and rapid method for calculating identity-by-descent matrices using multiple markers, Genet. Sel. Evol. 33 (2001) 453-471.

[20] Quaas R.L., Additive genetic model with groups and relationships, J. Dairy Sci. 71 (1988) 1338-1345.

[21] Quaas R.L., Anderson R.D., Gilmour A.R., BLUP school handbook; use of mixed models for prediction and estimation of (co)variance components, Animal Breeding and Genetics Unit, University of New England, NSW 2351, Australia, 1984.

[22] Quaas R.L., Pollak E.J., Mixed model methodology for farm and ranch beef cattle testing programs, J. Anim. Sci. 51 (1980) 1277-1287.

[23] Thompson E.A., Two-locus and three-locus gene identity by descent in pedigrees, IMA J. Math. Appl. Med. Biol. 5 (1988) 261-279.

[24] Tier B., Computing inbreeding coefficients quickly, Genet. Sel. Evol. 22 (1990) 419-430.

[25] Totir L.R., Fernando R.L., The effect of linkage on the additive by additive covariance between relatives, Genet. Sel. Evol. 30 (1998) 409-421. 
[26] Totir L.R., Fernando R.L., Dekkers J.C.M., Effect of using approximate gametic variance covariance matrices on marker assisted selection by BLUP, J. Anim. Sci. 80 (Suppl. 2) (2002) 20.

[27] van Arendonk J.A.M., Tier B., Kinghorn B.P., Use of multiple genetic markers in prediction of breeding values, Genetics 137 (1994) 319-329.

[28] Wang T., van der Beek S., Fernando R.L., Grossman M., Covariance between effects of marked QTL alleles, J. Anim. Sci. 69 (Suppl. 1) (1991) 202.

[29] Wang T., Fernando R.L., Grossman M., Genetic evaluation by BLUP using marker and trait information in a multibreed population, Genetics 148 (1998) 507-515.

[30] Wang T., Fernando R.L., van der Beek S., Grossman M., van Arendonk J.A.M., Covariance between relatives for a marked quantitative trait locus, Theor. Appl. Genet. 27 (1995) 251-274.

To access this journal online: www.edpsciences.org 